\title{
Three-Dimensional Magnetic Page Memory
}

\author{
O. Ozatay, ${ }^{1,{ }^{*}}$ A. Gokce, ${ }^{1}$ T. Hauet, ${ }^{2}$ L. Folks, ${ }^{3}$ A. Giordano, ${ }^{4}$ and G. Finocchio ${ }^{4}$ \\ ${ }^{1}$ Physics Department, Bogazici University, 34342 Bebek, Istanbul, Turkey \\ ${ }^{2}$ Institut Jean Lamour, UMR CNRS-Université de Lorraine, 54011 Nancy, France \\ ${ }^{3}$ Department of Electrical Engineering, School of Engineering and Applied Sciences, University at Buffalo, \\ Buffalo, New York 14260, USA \\ ${ }^{4}$ Department of Mathematical and Computer Sciences, Physical Sciences and Earth Sciences, University of \\ Messina, Messina I-98166, Italy
}

(Received 26 March 2018; revised manuscript received 25 July 2018; published 2 January 2019)

\begin{abstract}
The increasing need to store large amounts of information with an ultradense, reliable, low-power, and low-cost memory device is driving aggressive efforts to improve on current perpendicular magnetic recording technology. However, the difficulties in fabricating small-grain recording media while maintaining thermal stability and a high signal-to-noise ratio motivate the development of alternative methods, such as the patterning of magnetic nanoislands and use of energy assistance for future applications. In addition, from both a sensor perspective and a memory perspective, three-dimensional spintronic devices are highly desirable to overcome the restrictions on the functionality in planar structures. Here we demonstrate a three-dimensional magnetic memory (magnetic page memory) based on thermally assisted and strayfield-induced transfer of domains in a vertical stack of magnetic nanowires with perpendicular anisotropy. Use of spin-torque-induced domain multiplication in such a device with periodic pinning sites provides additional degrees of freedom by allowing lateral information flow to realize truly-three-dimensional integration.
\end{abstract}

DOI: 10.1103/PhysRevApplied.11.014002

\section{INTRODUCTION}

The application of instantaneous heat pulses to achieve brief periods of temperature increase in a recording medium results in sudden changes in a number of physical properties that can be exploited in a wide spectrum of data-storage applications. This approach is already used in optical recording, such as compact discs and digital versatile discs, where the heat source is a laser pulse that causes a change in the crystallinity of the chalcogenide recording medium, leading to marked modifications of the optical reflectivity [1]. If the laser heat source is replaced by resistive heating from electrical pulses and the physical property being monitored is the electrical resistivity of the chalcogenide material, then the resulting device (phase-change memory) can be used in storage-class memory applications $[2,3]$. In magnetic recording, to overcome the limitations in scaling imposed by the thermal stability of small grains [4], thermally assisted recording with a laser beam coupled to a near-field transducer as the heat source instantaneously reduces the reversal fields required to write to high-anisotropy media, allowing thermally stable grain sizes down to a few nanometers $[5,6]$.

\footnotetext{
*ozhan.ozatay@boun.edu.tr
}

Even though the local injection of electrical current pulses with a scanning-tunneling-microscope tip has been shown to be an effective way to induce local heating to allow the manipulation of magnetization at the nanoscale [7], a magnetic-memory-device concept taking advantage of such local heating due to electrical pulses has not yet been realized.

Here we use the local heating from electrical pulses in a three-dimensional magnetic memory device [8] (magnetic page memory) that comprises an array of magnetic nanowires with perpendicular magnetic anisotropy, stacked in a cross-wire configuration where individual layers of nanowires are electrically isolated with a lowthermal-conductivity silicon nitride spacer [9] as shown in Figs. 1(a) and 1(b). Such a memory design with threedimensional storage capability is very desirable from a technology point of view [10-12] since it is expected to provide a low-cost-per-bit, high-speed, and ultradense memory solution. In addition, such three-dimensional magnetic memory does not suffer from loss of information in the long run due to resistance drift in phase-change memory [3] and charge leakage in flash memory. This high potential inspired the industry to investigate alternative approaches such as three-dimensional magneticdomain-wall racetrack memory [13-15] based on the 


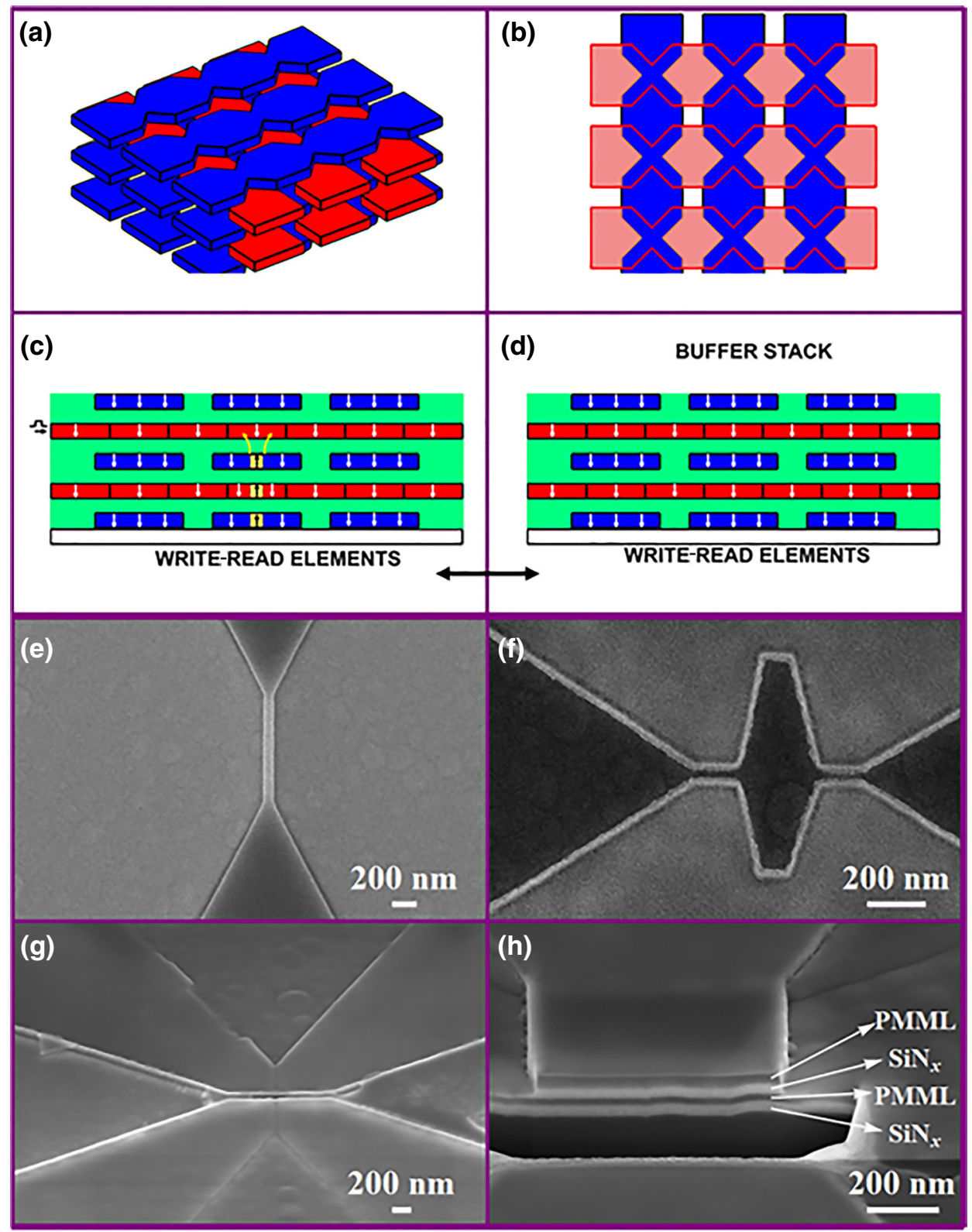

FIG. 1. Magnetic page memory: principle of operation. (a) Three-dimensional schematic of the perspective view of an array of nominally identical, perpendicular-anisotropy magnetic nanowires with periodic nucleation/pinning sites stacked in a cross-wire configuration. Alternating layer of nanowires are colored blue and red. (b) Top-view schematic of the nanowire stack displaying the vertical alignment of pinning sites acting as storage bits in the cross-wire architecture. Alternating layer of nanowires are colored blue and red. (c),(d) Side view schematic of the page memory device with a single layer of read/write elements, a stack of nanowire arrays with perpendicular magnetization separated by a silicon nitride low-thermal-conductivity spacer (green region), where the straight arrows represent the local magnetization direction and the curved arrows represent the local stray field. A square electrical pulse is applied to the layer to be written to so as to instantaneously lower its coercivity. The buffer stack in (d) is a replica of the memory array that is used for temporary storage in the read process. (e)-(h) Scanning-electron-microscopy images of CoNi-Pd multilayer nanowires: (e) single nanowire $100 \mathrm{~nm}$ wide and $1 \mu \mathrm{m}$ long, designed for writing experiments; (f) a nanowire with a double-constriction designed for spin-torque-induced lateral-domain-transfer studies; (g) two nanowires with a 20-nm-thick silicon nitride spacer in the cross-wire configuration designed to study the heat-assisted vertical domain transfer in page memory; (h) a focused-ion-beam cut of the sample shown in $(\mathrm{g})$ showing the film structure consisting of perpendicular-anisotropy magnetic multilayers (PMML) separated by a silicon nitride spacer. 
interaction of a spin-polarized current pulse with a magnetic domain wall [16]. However, the nanofabrication challenges involved in the actual realization of an array of vertical racetracks with periodic pinning sites required for the three-dimensional geometry present significant obstacles [14].

\section{OPERATION PRINCIPLE}

The basic operation principle of a magnetic-pagememory device is shown in Figs. 1(a)-1(d). A perpendicular-anisotropy-magnetic-nanowire array with periodic domain nucleation sites (constrictions) in a cross-wire architecture is built on a single layer of read-write elements. After all the data have been recorded on the first magnetic layer in the form of the presence or absence of domains in the nanowire constrictions, the domain configuration is replicated in the next layer by instantaneous reduction of the nucleation fields through local Joule heating from electrical pulses. The increasing temperature in the nucleation sites of the second layer makes its local magnetization susceptible to the stray fields coming from the recorded domains in the first magnetic layer. The thirdmagnetic-layer constrictions are also heated synchronously with the second magnetic layer so that the stray field coming from the top layer is suppressed. After the electrical pulses are turned off, the second-layer domain configuration ends up being a replica of the first magnetic layer. The replication of the information recorded in the second magnetic layer to the layer above it proceeds in a similar manner through sequential heating of the third and fourth magnetic layers and cooling in the presence of the stray fields available from the perpendicularly oriented domains as shown in Fig. 1(c).

To reach a data point recorded in a given layer, a buffer stack [Fig. 1(d)] is used to maintain the information stored beneath the layer of interest. For instance, to read data from the second layer, the information in the first layer is read and transferred by the read-write elements to the first layer of a buffer stack. The read element can, for instance, be a conventional tunneling-magnetoresistance head and the write element could be a conventional perpendicular write head already used in perpendicular magnetic recording heads [8]. Then by application of electrical pulses in the same manner as above, the first layer replicates the domain structure of the second layer, making the data accessible to the read-write elements. Here the insulating-spacer thickness is chosen to ensure both magnetic transparency and thermal isolation [17-20] between layers to avoid disturbing the magnetization configuration of neighboring layers [21]. The periodic constrictions where the domains nucleate also act as pinning sites both to fix the position of the information (domains) and to enable highly concentrated current densities that give rise to the localized heating process for the vertical domain-transfer process.

\section{EXPERIMENTAL SETUP}

The different device types we study to demonstrate the page-memory concept are summarized in Figs. 1(e)-1(h). We use magnetic nanowires with a composition of $\mathrm{Ta}(1.5 \mathrm{~nm}) / \mathrm{Pd}(3 \mathrm{~nm}) /\left[\mathrm{Co}_{55} \mathrm{Ni}_{45}(0.22 \mathrm{~nm}) / \mathrm{Pd}(1.2\right.$ $\mathrm{nm})] \times 22$ repeats $/ \operatorname{Pd}(2 \mathrm{~nm})$. The single-layer singlenanowire structures [Fig. 1(e)] serve to characterize the writing conditions [21]. The double-constriction samples [Fig. 1(f)] enable the study of lateral domain transfer by use of spin-transfer torque [22]. In the double-layer version [Fig. 1(g)] the individual layers of nanowires in the crosswire architecture are isolated with a 20-, 40-, or 60-nmthick silicon nitride spacer as the low-thermal-conductivity spacer. Figure 1(h) shows a scanning-electron-microscope image of a focused-ion-beam cut of the double-layer structure in Fig. 1(g). The spacer layer sandwiched between perpendicular-anisotropy magnetic multilayer nanowires plays several crucial roles in the operation of such a device. It not only isolates the individual layers electrically and thermally but also ensures magnetic transparency, which can be controlled with a proper choice of the film thickness.

A simple schematic drawing of the experimental setup is shown in Fig. 2. For the purpose of this experiment, a commercial magnetic-force-microscope (MFM) tip (Bruker MESP-HM) placed a few nanometers above the surface of the top nanowire is used as the source of stray fields
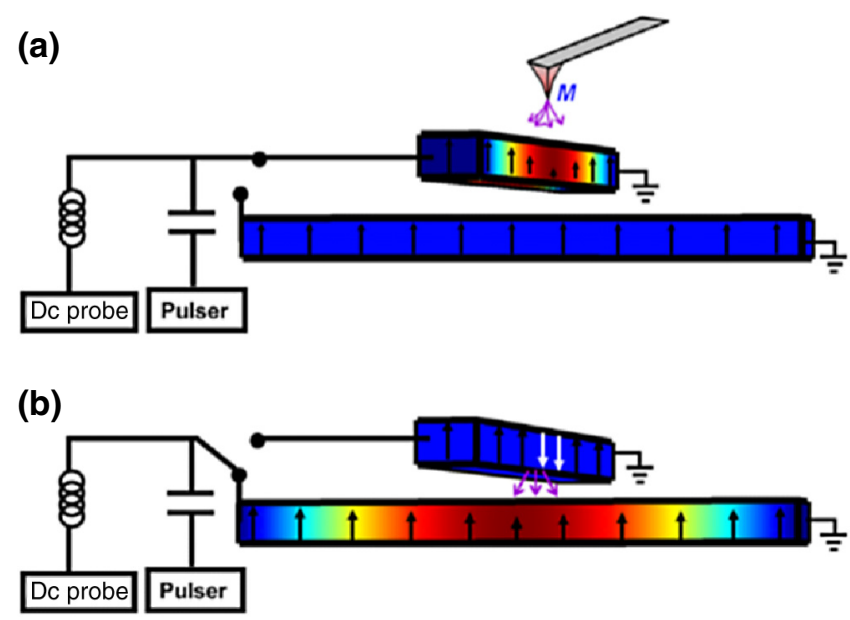

FIG. 2. Schematics of the experimental setup. (a) Heat-assisted writing using a magnetic-force-microscope tip placed a few nanometers above the surface of the top nanowire as the source of stray fields. The bottom nanowire is grounded, whereas the resistance of the top nanowire is monitored by a dc probe before and after the application of electrical pulses. (b) The top-nanowire reversal domain acts as the source of stray fields to write onto the bottom nanowire, which is made susceptible to such fields through resistive heating from electrical pulses. The dc probe monitors the resistance of the bottom nanowire before and after the application of pulses, whereas the top nanowire is grounded. 
to induce magnetization reversal locally in the nanowire underneath. At the tip of the cantilever the magnetization is forced to point out of plane due to the shape anisotropy, and stray fields as high as 700 Oe are available from such tips [23]. The measured bulk nucleation field of our

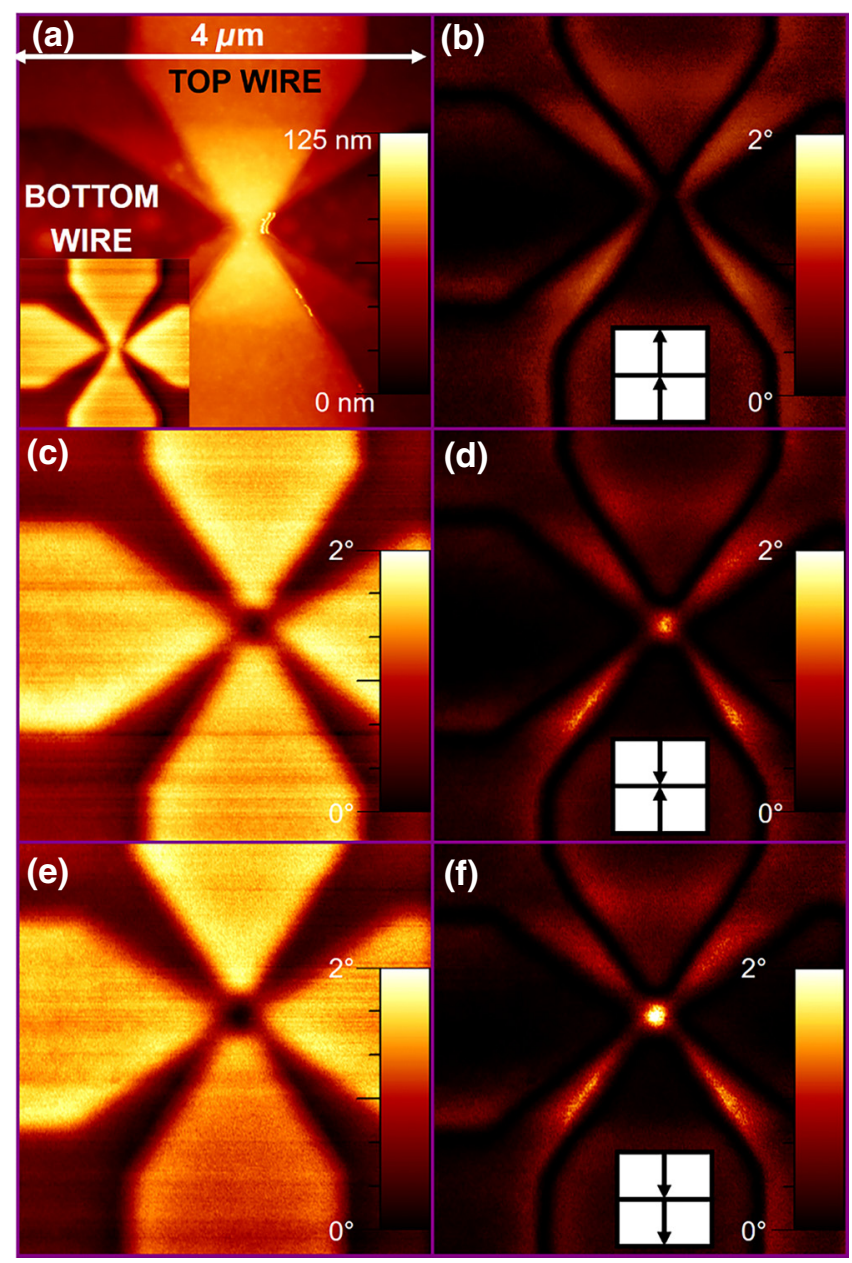

FIG. 3. Experimental detection of the vertical heat-assisted domain transfer. (a) Atomic microscope image of the topography. The inset shows a MFM image of the out-of-plane remanent state after saturation in a 2-T out-of-plane magnetic field in a single 100-nm-wide constriction sample. (b) The MFM image in the inset in (a) multiplied by itself (all the pixel intensities are squared) and replotted on the same color scale as in (d) and (f). Top- and bottom-constriction magnetizations pointing out of plane. (c) MFM image after the application of a 500-mV, 10-ns pulse to the top constriction in the presence of a magnetic tip parked $1 \mathrm{~nm}$ above the surface. (d) The MFM image in (c) multiplied by itself (all the pixel intensities are squared) and replotted on the same color scale as in (b) and (f). Top-constriction magnetization reversed. (e) MFM image after the application of a $500-\mathrm{mV}, 10-\mathrm{ns}$ pulse to the bottom constriction in the presence of the stray field from the top nanoconstriction. (f) The MFM image in (e) multiplied by itself (all the pixel intensities are squared) and replotted on the same color scale as in (b) and (d). Bottom-constriction magnetization reversed. film structure is approximately $3 \mathrm{kOe}$, much higher than the stray fields available from MESP-HM magnetic tips. Therefore, at room temperature the magnetic tip is not able to trigger magnetization reversal. However, if an electrical pulse sufficiently high in amplitude (approximately $3 \times 10^{7} \mathrm{~A} / \mathrm{cm}^{2}$ ) and width (approximately $8 \mathrm{~ns}$ ) is applied to the nanowire, the local nucleation field is reduced due to increasing temperature from current-induced resistive heating [21]. By our grounding the bottom nanowire during the pulse application, only the top nanowire is made to be susceptible to the stray field-induced reversal as shown in Fig. 2(a). A dc probe independently (through a bias tee) monitors the dc resistance of the top nanowire to detect magnetoresistance effects due to domain nucleation. To transfer the top domain to the bottom nanowire the measurement setup is reconnected to the bottom nanowire and the perpendicularly oriented domain at the top nanowire is used as a source of the stray field (the magnetic tip is removed). Analogous to the former case the bottom nanowire is heated with an electrical pulse and its magnetization is made susceptible to reversal due to the stray field [Fig. 2(b)].
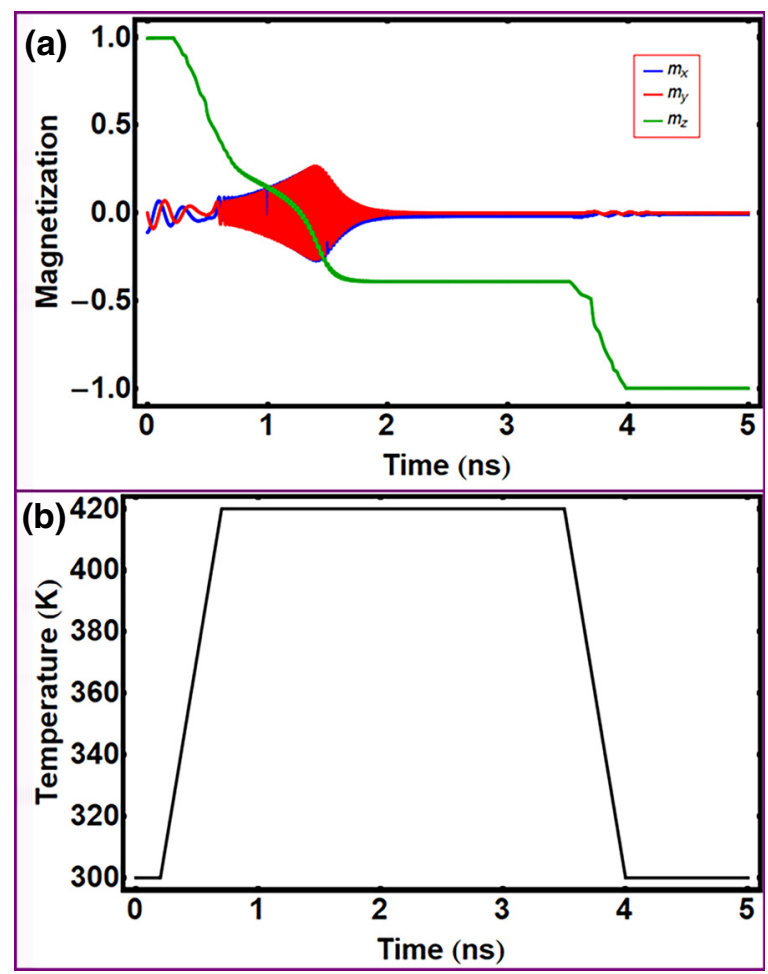

FIG. 4. Macrospin Landau-Lifshitz-Bloch simulation results. (a) Time-domain traces of the magnetization switching $\left(m_{z}\right.$ switches from +1 to -1 , black line) computed with the LandauLifshitz-Bloch equation within the macrospin approximation as a response to the thermal pulse. (b) Temperature change with time. The ferromagnet is biased with an external field that is of the same order of the dipolar field that can originate from the bottom layer $(-30 \mathrm{mT})$. 


\section{RESULTS}

The domain configurations for the remnant state (after the application of a 2-T out-of-plane field) [Fig. 3(a), inset], the state after magnetic writing (with a $500-\mathrm{mV}$, 10-ns-wide pulse) to the top nanowire with a MFM tip [Fig. 3(c)], and the state after domain replication from the top nanowire to the bottom nanowire [Fig. 3(e)] can be directly imaged with magnetic force microscopy, provided that the magnetic signal from the bottom nanowire has a significant contribution to the image contrast. Figure 3(a) shows the corresponding topography image. The bottom wire lies horizontally, whereas the top wire lies vertically. The 100-nm-wide constrictions acting as pinning sites are aligned on top of each other. To distinguish the two cases when there is a reversal domain in the top nanowire and in both nanowires, the images shown in the inset in Fig. 3(a) and in Figs. 3(c) and 3(d) are multiplied by themselves and replotted with inverted colors as shown in Figs. 3(b), 3(d), and 3(f), respectively (i.e., the MFM signal intensity in each pixel is squared). When the resulting images are plotted on the same color scale with inverted contrast, the relative contribution to the MFM signal from the bottom nanowire is visually amplified [appears brighter as in Fig. 3(f)]. We find that this enhancement of the MFM signal (approximately 30\%) is accompanied by a reversible change in the resistance (approximately $90 \Omega$ ) of the bottom nanowire (approximately $90 \mathrm{~m} \Omega \leq 0.1 \%$ ) as detected by the dc probe. Such small magnetoresistance effects due to constricted domain walls have been observed previously [24] and have been ascribed to domain-wall anisotropic magnetoresistance [25]. This writing process can be clearly understood from macrospin simulations based on the Landau-LifshitzBloch equation [26,27]. Figure 4(a) shows an example of time-domain evolution of the three components of the magnetization $\left(m_{x}, m_{y}\right.$, and $\left.m_{z}\right)$ for a thermally assisted stray-field-induced writing as a response to a thermal pulse $(420 \mathrm{~K})$ and for a bias field of $\mathbf{H}_{\text {appl }}=-30 \mathrm{mT}$. This pulse is due to the Joule heating induced by the current flowing shown in Fig. 4(b), while the dipolar interactions originate from the bottom ferromagnetic layer, considered with the magnetization vector pointing along the $-z$ direction [21].

The ability to perform spin-polarized current-induced, intralayer horizontal translation of domains, in addition to the vertical interlayer replication, in a page-memory device would enable truly-three-dimensional dynamic information flow. Figure 5 shows the possible outcomes of the interaction between a spin-polarized current pulse and a written domain in a double-constriction sample (with

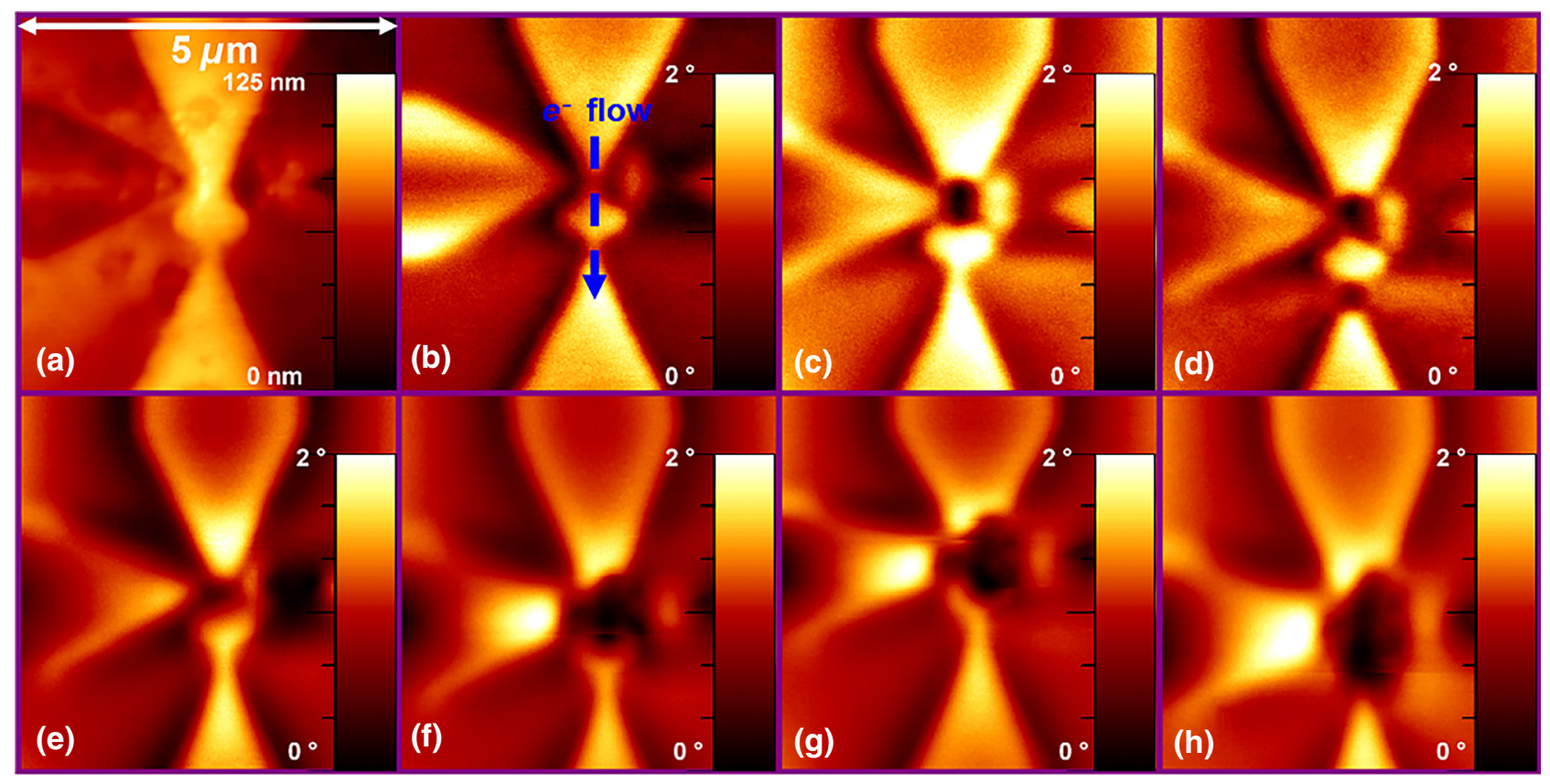

FIG. 5. Experimental detection of the spin-torque-induced lateral domain transfer. (a) Atomic force microscope image of a doubleconstriction (200 nm top-100 nm bottom) sample with 500-nm lateral spacing between the constrictions. (b) MFM image of the remanent state after saturation with a 2-T out-of-plane field. The blue arrow represents the electron flow direction for pulsing experiments. (c) MFM image after the application of a $800-\mathrm{mV}, 10-\mathrm{ns}$ pulse with a magnetic tip placed $1 \mathrm{~nm}$ above the surface of the top constriction. (d) MFM image after the application of a 2.2-V, 1-ns pulse in the absence of the magnetic tip. (e) MFM image after resaturation and rewriting of the reversal domain in the top constriction. (f) MFM image after the application of 1-V, 1-ns pulses five times in the absence of the magnetic tip. (g) MFM image after the application of two additional 1-V, 1-ns pulses in the absence of the magnetic tip. (h) MFM image after the application of five additional 1-V, 1-ns pulses in the absence of the magnetic tip. 
200- and $100-\mathrm{nm}$-wide constrictions). After saturating the magnetization out of plane with a 2-T magnetic field [Fig. 5(b)], at remanence we apply an 800-mV-amplitude, 10 -ns-wide pulse when a magnetic tip is parked $1 \mathrm{~nm}$ above the surface of the top constriction. Figure 5(c) shows that the top-constriction magnetization is reversed as a result of the local heating and the stray field from the tip. When the tip is removed and an additional electrical pulse (with electron flow from top to bottom) of $2.2-\mathrm{V}$ amplitude and 1-ns width is applied (corresponding to $1 \times 10^{8} \mathrm{~A} / \mathrm{cm}^{2}$ ), we find that the top domain is replicated at the bottom constriction without the reversal of the intermediate region between the two constrictions [Fig. 5(d)]. However, if the same experiment is performed with $1-\mathrm{V}$, 1 -ns $\left(4.5 \times 10^{7} \mathrm{~A} / \mathrm{cm}^{2}\right)$ pulses, then the written domain grows into the intermediate region between the constrictions until it is finally captured at the bottom constriction after the application of 12 pulses [Figs. 5(e)-5(h)]. The gradual propagation of the domain wall into the spacer does not appear to be isotropic [Fig. 5(g)] and finds stable pinning points as the domain wall hits the corners od the hexagonal spacer region.

These two distinct regimes of the interaction between the domain walls and the spin-polarized current in this system can be understood with micromagnetic [28] modeling [21] taking into account the spatial variations of magnetic properties due to current-induced spatially inhomogeneous heating (for details of the simulation parameters, see Supplemental Material [21]). In these simulations $[29,30]$ we assume that the primary effect of the different temperatures (in addition to change of anisotropy and magnetization) in the two cases shown in Fig. 6 is to change the effective anisotropy direction so that lower current densities result in more out-of-plane magnetization, whereas high-current densities cause enough heating to have shape anisotropy pull the effective magnetization direction in plane. Figures 6(a)-6(d) show the case of in-plane spin-transfer torque that causes domain expansion into the spacer region without the corners being touched [17,31]. When the current is turned off [Fig. 6(d)], the stable domain configuration is that of a replication of the reversal region in the second constriction, similar to the situation shown in Figs. 5(c) and 5(d). However, if the effective anisotropy points dominantly out of plane, we find that the domain propagation into the spacer is gradual, and even instantaneous decoupling of domains may occur. When the current is turned off, the stable configuration is one where the contraction of the domain back to the initial state is hindered by the sharp corners acting as pinning sites in the spacer region in Figs. 6(e)-6(h) similarly to the case in Figs. 5(e)-5(h). Therefore, the use of spin-polarized current pulses results in either domain expansion to or domain replication in the next constriction along the direction of electron flow depending on the

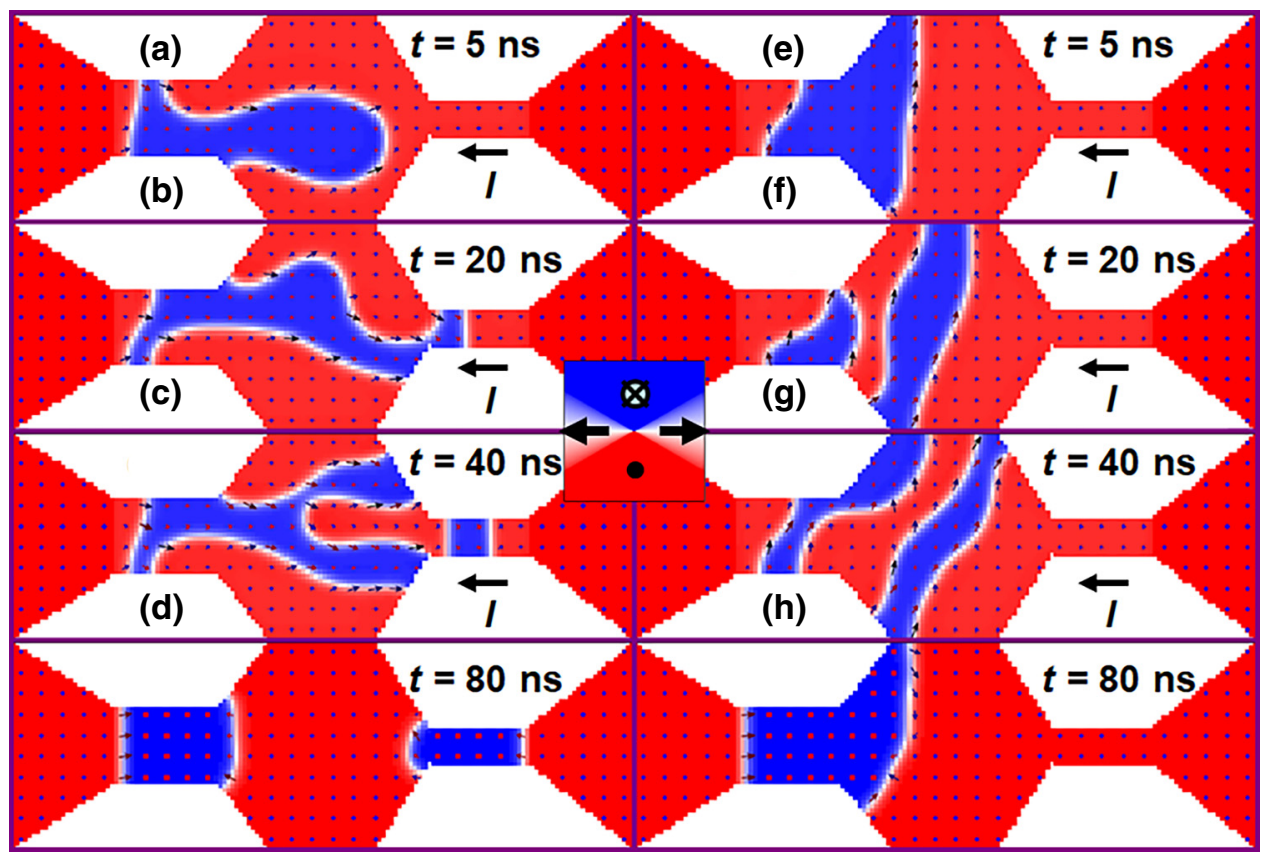

FIG. 6. Micromagnetic simulations of Lateral domain transfer. (a)-(c) The micromagnetic configuration of a double-constriction sample (200 and $100 \mathrm{~nm}$ ) at 5, 20, and $40 \mathrm{~ns}$ after a 4-mA electrical current is turned on. (d) The micromagnetic configuration $30 \mathrm{~ns}$ after the current is turned off. (e)-(g) The micromagnetic configuration of a double-constriction sample (200 and $100 \mathrm{~nm})$ at 5, 20, and $40 \mathrm{~ns}$ after a 4-mA electrical current is turned on assuming an out-of-plane polarization. (h) The micromagnetic configuration $30 \mathrm{~ns}$ after the current is turned off. The color scale represents the $z$ component of the magnetization as shown in the center, and the arrows represent the local magnetization direction. 
effective spin-polarization direction. This implies that in a magnetic-page-memory device, use of only one read-write element per nanowire in conjunction with spin-polarized current pulses to propagate the information is sufficient to generate the first storage layer, which can be vertically replicated to the next layer on top. This domain injection is qualitatively similar to blowing of magnetic skyrmion bubbles [32].

In conclusion, we demonstrate the operation of a threedimensional magnetic-page-memory device that takes advantage of a combination of vertical data flow using local heating from current pulses in the presence of perpendicular stray fields and intralayer horizontal flow using spin-polarized current pulses. Such an approach could lead to a memory solution fully utilizing three-dimensional space with ultradense data-storage capacity. The current work on the three-dimensional magnetic page memory could also be potentially beneficial for future applications of newly discovered complex magnetic nanostructures such as magnetic skyrmions, domain walls, and solitons, which could replace current magnetic bits with potentially higher mobilities due to interactions with spin-polarized currents as well as the spin-orbit torque from pure spin currents.

\section{ACKNOWLEDGMENTS}

This work was supported in part by the Bogazici University Research Fund under Grant No. 16B03P5, the Scientific and Technological Research Council of Turkey (TUBITAK) under Contract No. 112T205, the French PIA project "Lorraine Université d'Excellence" (ANR-15IDEX-04-LUE), and the CNRS and TUBITAK "PICS" program (2016). The authors acknowledge the help of Dr. Andreas Moser for magnetic-force-microscopy imaging and analysis, Dr. Jordan A. Katine for device nanofabrication, Dr. Jan-Ulrich Thiele for optimization of materials, Dr. Sylvia H. Florez for magnetic characterization, and Dr. Bruce D. Terris for interpretation of the data.

[1] H. Coufal and L. Dhar, Materials for optical data storage, MRS Bull. 31, 294 (2006).

[2] O. Ozatay, B. Stipe, J. A. Katine, and B. D. Terris, Electrical switching dynamics in circular and rectangular Ge2Sb2Te5 nanopillar phase change memory devices, J. Appl. Phys. 104, 084507 (2008).

[3] A. Gokce, I. Cinar, S. C. Ozdemir, E. Cogulu, B. Stipe, J. A. Katine, and O. Ozatay, Toward multiple-bit-per-cell memory operation with stable resistance levels in phase change nanodevices, IEEE Trans. Electron Devices 63, 3103 (2016).

[4] D. Weller and A. Moser, Thermal effect limits in ultra-high density magnetic recording, IEEE Trans. Magn. 35, 4423 (1999).
[5] M. Kryder, E. C. Gage, T. W. Mcdaniel, W. A. Challener, R. E. Rottmayer, G. P. Ju, Y. T. Hsia, and M. F. Erden, Heat assisted magnetic recording, Proc. IEEE 96, 1810 (2008).

[6] B. C. Stipe, T. C. Strand, C. C. Poon, H. Balamane, T. D. Boone, J. A. Katine, J. L. Li, V. Rawat, H. Nemoto, A. Hirotsune, O. Hellwig, R. Ruiz, E. Dobisz, D. S. Kercher, N. Robertson, T. R. Albrecht, and B. D. Terris, Magnetic recording at $1.5 \mathrm{Pbm}-2$ using an integrated plasmonic antenna, Nat. Photonics 4, 484 (2010).

[7] L. Zhang, J. A. Bain, J.-G. Zhu, L. Abelmann, and T. Onoue, Characterization of heat-assisted magnetic probe recording on $\mathrm{CoNi} / \mathrm{Pt}$ multilayers, J. Magn. Magn. Mater. 305, 16 (2006).

[8] O. Ozatay, and B. D. Terris, Read/write structures for a three dimensional memory, US 2011141792 A1 (United States Patent and Trademark Office, 24 April 2012).

[9] R. E. Fontana, A. Moser, H. J. Rosen, B. D. Terris, C. H. Tsang, Three-dimensional Magnetic Memory, US 20080205116 A1 (United States Patent and Trademark Office, 20 October 2009).

[10] S. L. Zhang, J. Y. Zhang, A. A. Baker, S. G. Wang, G. H. $\mathrm{Yu}$, and T. Hesjedal, Three dimensional magnetic abacus memory, Sci. Rep. 4, 6109 (2014).

[11] R. Lavrijsen, J. H. Lee, A. Fernandez-Pacheco, D. C. M. C. Petit, R. Mansell, and R. P. Cowburn, Magnetic ratchet for three-dimensional spintronic memory and logic, Nature 493, 647 (2013).

[12] A. Fernandez-Pacheco, R. Streubel, O. Fruchart, R. Hertel, P. Fischer, and R. P. Cowburn, Three-dimensional nanomagnetism, Nat. Commun. 8, 15756 (2017).

[13] S. S. P. Parkin, Shiftable magnetic shift register and method of using the same, US 6834005, System and method for writing to a magnetic shift register, US 6898132, System and method for reading data stored on a magnetic shift register, US 6920062, Magnetic shift register with shiftable magnetic domains between two regions, and method of using the same, US 7031178, and System and method for transferring data to and from a magnetic shift register with a shiftable data column, US 7236386, United States Patent and Trademark Office (2004-2007).

[14] S. S. P. Parkin, M. Hayashi, and L. Thomas, Magnetic domain-wall racetrack memory, Science 320, 190 (2008).

[15] S. S. P. Parkin and S. H. Yang, Memory on the racetrack, Nat. Nanotechnol. 10, 195 (2015).

[16] L. Berger, Exchange interaction between ferromagnetic domain-wall and electric-current in very thin metallicfilms, J. Appl. Phys. 55, 1954 (1984).

[17] J. P. Feser, J. Liu, and D. G. Cahill, Pump-probe measurements of the thermal conductivity tensor for materials lacking in-plane symmetry, Rev. Sci. Instrum. 85, 104903 (2014).

[18] H. K. Lyeo and D. G. Cahill, Thermal conductance of interfaces between highly dissimilar materials, Phys. Rev. B 73, 144301 (2006).

[19] E. T. Swartz and R. O. Pohl, Thermal boundary resistance, Rev. Mod. Phys. 61, 605 (1989).

[20] O. Ozatay, T. Hauet, S. H. Florez, J. A. Katine, A. Moser, J. U. Thiele, L. Folks, and B. D. Terris, Probing activation energy barrier distribution for reversal of strongly 
exchange-coupled magnetic multilayer thin films, Appl. Phys. Lett. 95, 172502 (2009).

[21] See Supplemental Material at http://link.aps.org/supple mental/10.1103/PhysRevApplied.11.014002 for details on sample fabrication, sample characterization, and additional finite-element and micromagnetic simulation data.

[22] Z. Li and S. Zhang, Magnetization dynamics with a spintransfer torque, Phys. Rev. B 68, 024404 (2003).

[23] M. Jaafar, A. Asenjo, and M. Vazquez, Calibration of Coercive and Stray Fields of commercial magnetic force microscope probes, IEEE Trans. Nanotechol. 7, 245 (2008).

[24] O. Ozatay, P. Chalsani, N. C. Emley, I. N. Krivorotov, and R. A. Buhrman, Magnetoresistance and magnetostriction effects in ballistic ferromagnetic nanoconstrictions, J. Appl. Phys. 95, 7315 (2004).

[25] C. H. Marrows, Spin-polarized currents and magnetic domain-walls, Adv. Phys. 54, 585 (2005).

[26] U. Kilic, G. Finocchio, T. Hauet, S. H. Florez, G. Aktas, and O. Ozatay, Magnetic switching driven by nanosecond scale heat and magnetic field pulses: An application of macrospin Landau-Lifshitz-Bloch model, Appl. Phys. Lett. 101, 252407 (2012).
[27] U. Atxitia, D. Hinzke, and U. Nowak, Fundamentals and applications of the Landau-Lifshitz-Bloch equation, J. Phys. D: Appl. Phys. 50, 033003 (2017).

[28] NIST technical report: OOMMF User's Guide, Version 1.0 M.J. Donahue and D.G. Porter Interagency Report NISTIR 6376 (National Institute of Standards and Technology, Gaithersburg, MD, 1999).

[29] G. Finocchio, B. Azzerboni, G. D. Fuchs, R. A. Buhrman, and L. Torres, Micromagnetic modeling of magnetization switching driven by spin-polarized current in magnetic tunnel junctions, J. Appl. Phys. 101, 063914 (2007).

[30] M. Carpentieri, R. Tomasello, S. Vergura, F. Garesci, G. Siracusano, M. d'Aquino, and G. Finocchio, Micromagnetic analysis of statistical switching in perpendicular magnetic tunnel junctions with double reference layers, IEEE Magn. Lett. 9, 3102105 (2018).

[31] D. Claudio-Gonzalez, A. Thiaville, and J. Miltat, Domain Wall Dynamics under Nonlocal Spin-Transfer Torque, Phys. Rev. Lett. 108, 227208 (2012).

[32] W. J. Jiang, P. Upadhyaya, W. Zhang, G. Q. Yu, M. B. Jungfleisch, F. Y. Fradin, J. E. Pearson, Y. Tserkovnyak, K. L. Wang, O. Heinonen, S. G. E. te Velthuis, and A. Hoffmann, Blowing magnetic skyrmion bubbles, Science 349, 283 (2015). 